\title{
Visión de la extensión en Sedes Interuniversitarias, recomendaciones para la incorporación de acciones en zonas regionales. Eje: políticas de extensión. Sub-eje: orientaciones para las políticas de extensión en la Universidad Nacional
}

A Vision of the University Extension in Inter-university Campuses: Recommendations to Integrate Activities in Regional Areas. Core Topic: Extension

Policies. Subtopic: Guidelines for the University Extension Policies at National University of Costa Rica

Karla Miranda Benavides Universidad Nacional Alajuela, Costa Rica kmiranda@una.cr

Gaudy Prendas Aguilar Universidad Nacional Alajuela, Costa Rica prendas.gaudy@gmail.com

Scatk Noguera Moya Universidad Nacional Alajuela, Costa Rica scatk.noguera.moya@una.cr

Recibido 20/01/2018 Aceptado 30/03/2018 
Revista Universidad en DiÁlogo • Vol. 8, N. ${ }^{\circ}$ 1, Enero-Junio, 2018, pp. 57-71

ISSN 2215-2849 • EISSN: 2215-4752

DOI: http://dx.doi.org/10.15359/udre.8-1.4

Resumen. El propósito de creación de las Sedes Interuniversitarias es desarrollar la acción sustantiva de la Universidad Nacional con participación de otras universidades públicas, lo cual representa una gran oportunidad para trasladar los resultados de la acción docente a las comunidades de las diversas zonas regionales. Este artículo expone la visión de un grupo de académicos en torno a la extensión universitaria en el ámbito de las Sedes; se describe el estado de la extensión en los planes de estudio de la Sede Interuniversitaria de Alajuela y se presenta un análisis de la normativa vigente respecto a la extensión. Además, se estudia un modelo de análisis administrativo y su aplicación en términos de una política de extensión. También se expone la importancia del trabajo con las comunidades en el ámbito de los programas de los cursos; tarea de la cual se desprenden recomendaciones para la ejecución de la extensión universitaria.

Palabras clave: extensión universitaria, sociedad, políticas

\begin{abstract}
The purpose of creating Sedes Interuniversitarias (Inter-university Campuses) is to develop the substantive university action with the participation of public universities in Costa Rica; this represents a great opportunity to transfer the results of the teaching action to the communities in different regional areas. This article presents the vision of a group of professors on the university extension in Sedes Interuniversitarias. It also describes the university extension status in the study programs at Sedes. Moreover, it includes an analysis of the current regulations regarding university extension, as well as a model of administrative analysis and its application in terms of an extension policy. Finally, the article addresses the importance of working with the communities as part of the study programs; from this, recommendations are proposed to better execute the university extension.
\end{abstract}

Keywords: university extension, society, politics.

\title{
Justificación
}

A partir de la creación de la Sede Interuniversitaria de Alajuela en el año 2007, mediante acuerdo de CONARE CNR-106-07, se establece como acuerdo: "b. Desarrollar una oferta coordinada de oportunidades docentes, de investigación y de extensión ampliando el marco de atención en las distintas comunidades de la República, especialmente impartiendo carreras y programas conjuntos que respondan a principios de pertinencia, impacto, cobertura, equidad y calidad". 
En el año 2015, la Universidad Nacional publica la actualización del Estatuto Orgánico donde se ratifica el involucramiento de la extensión como un ejercicio en la formación de los estudiantes y como dinámica docente en el capítulo I, artículo 6, donde se especifica que "la acción sustantiva de la universidad se realiza mediante la docencia, la investigación, la extensión, la producción y otras formas que establezca la normativa institucional, las cuales se complementan y nutren mutuamente".

Desde su creación, la Sede Interuniversitaria de Alajuela ha ofrecido cuatro planes de estudio en coordinación con diferentes instancias académicas de la Universidad Nacional, a saber: Bachillerato en Inglés, Bachillerato en Administración, Bachillerato en Química Industrial y Bachillerato en Ingeniería en Sistemas de Información.

A la fecha, todos estos planes han desarrollado la docencia universitaria como acción primordial, sin embargo, diez años después de su fundación, formalmente no existen inscritos programas o proyectos de extensión en la Sede Interuniversitaria, lo cual se convierte en un reto para el personal académico para impulsar la extensión en el marco de la actividad docente, por medio de los aportes que los diferentes programas puedan ofrecer a las comunidades cercanas.

De acuerdo con el artículo 54 del Estatuto Orgánico de la Universidad Nacional sobre las funciones del Consejo de Facultad, Centro o Sede en los incisos que son de interés mencionar para el presente artículo se detalla:

a) Velar por la excelencia académica, procurando que la oferta responda a las políticas, a las prioridades institucionales y a las necesidades del país.

d) Integrar el quehacer académico de la facultad, centro o sede a partir de los programas, planes y proyectos que impulsen sus unidades académicas.

g) Aprobar los planes de capacitación, mejoramiento profesional, becas e incentivos para los funcionarios de la facultad, centro o sede.

m) Ejercer otras funciones que emanen de este estatuto y de otra normativa vigente. 
Estos puntos permiten evidenciar que la Sede cuenta con la normativa que sustenta la práctica de la extensión promoviendo el mejoramiento continuo de su personal académico y la integralidad de los programas de los cursos, actividades y prácticas que proyectan el crecimiento, así como el cumplimiento de uno de los pilares de la educación superior pública que promulga la Universidad Nacional: la extensión social.

Las Sedes Interuniversitarias tienen un papel crítico en el desarrollo de las zonas regionales; si bien la normativa institucional faculta la extensión universitaria bajo este régimen, la visión de la actividad docente es impulsar la extensión por medio de los programas de los cursos, que a su vez promuevan el acercamiento con las comunidades.

Este artículo también plantea algunas reflexiones y recomendaciones redactadas con la expectativa de que se conviertan en el mediano plazo en una realidad, previendo la construcción de un nuevo campus para la Sede Interuniversitaria, lo cual también es una oportunidad considerando la posible construcción de instalaciones universitarias en la zona de Desamparados de Alajuela.

\section{Objetivos}

a) Describir el estado de las acciones de extensión en los planes de estudio de la Universidad Nacional que se imparten en la Sede Interuniversitaria de Alajuela, con el propósito de documentar las experiencias generadas en este proyecto.

b) Analizar la normativa vigente respecto a la extensión universitaria en la Universidad Nacional para comprender los alcances de este pilar institucional y proponer recomendaciones en el marco de dicha normativa.

c) Estudiar un modelo de análisis administrativo y su aplicación en términos de una política de extensión que se pueda replicar a las universidades públicas involucradas en Sedes Interuniversitarias.

d) Plantear recomendaciones que favorezcan el desarrollo de la extensión en las Sedes Interuniversitarias, con el fin de crear el ámbito de acción para el acercamiento y desarrollo de las comunidades cercanas. 


\section{Conceptos generales y normativa vigente}

En la actualidad, debido a diferentes cambios sociales, económicos y políticos, así como a dos de las fuerzas más poderosas del mundo: la tecnología y los cambios ambientales, se fomenta a toda costa un desarrollo sostenible con miras a obtener utilidades para empresas con fines de lucro y generar un impacto positivo de universidades y organizaciones sin fines de lucro, buscando un equilibrio próspero en todos los sentidos. Donde el quehacer diario produzca un ganar a lo interno de la organización y un ganar para todos los actores que forman parte de esa relación económicosocial con la empresa.

Aspectos relevantes como la innovación, la globalización, la creatividad y la sostenibilidad son temas de interés en todos los ámbitos de la sociedad. La forma para enlazar la práctica académica y estos conceptos, cumpliendo la misión de la Universidad Nacional mediante uno de sus ejes principales: la extensión, es el principio de un proyecto como Sede Interuniversitaria de Alajuela, que busca proyectar al académico, sensibilizar su práctica e integración en los diferentes programas de cursos y fomentar unidades interdisciplinarias de profesionales que permitan generar un impacto en el desarrollo de comunidades locales y regionales, con la participación activa de la comunidad universitaria.

El artículo 9 del Estatuto Orgánico señala que las Sedes Interuniversitarias "son instancias académicas que desarrollan la acción sustantiva con participación de otras universidades públicas, nacionales o extranjeras, de acuerdo con los principios, valores y fines estatutarios, y siguiendo la normativa dispuesta por la Universidad Nacional y las otras universidades participantes" (2015, p. 24).

Actividades sustantivas relacionadas con la práctica de la extensión buscan integrar acciones innovadoras, sistemáticas y transformadoras, en la búsqueda de una proyección de universidad que integra no solo el saber, sino que suma el hacer y el querer hacer como una forma natural de ejecutar proyectos que tengan impacto, más allá de las aulas de enseñanza.

De igual manera, la extensión docente debe promoverse como una acción alineada con los procesos de acreditación, considerando los proyectos de extensión como evidencia básica para todos los programas de educación superior. 
Revista Universidad en DiÁlogo • Vol. 8, N. ${ }^{\circ}$ 1, Enero-Junio, 2018, pp. 57-71

ISSN 2215-2849 • EISSN: 2215-4752

DOI: http://dx.doi.org/10.15359/udre.8-1.4

Para ello se busca el planteamiento de una política de extensión, pero ¿cómo podemos definir el término política? Según Newston, J. (2011), una política organizacional se refiere a conductas intencionales para aumentar o proteger la influencia y el interés de una persona, sin dejar de inspirar confianza en las demás.

Establecer con claridad una política como Sede, en el sentido de fomentar de manera sistemática el modelo a seguir para el planteamiento y puesta en marcha de proyectos de extensión, es una necesidad latente, lista para plantear y llevarla a la práctica mediante la gestión adecuada a nivel interno de solicitud de fondos y el establecimiento adecuado de dichas prácticas con una misión y visión claras. Por ende, es necesario establecer una fórmula estratégica completa que permita brindar resultados que serán comparados con los objetivos planteados, permitiendo medir la efectividad de los proyectos en cuestión.

La Vicerrectoría de Docencia de la Universidad Nacional describe la gestión de proyectos de la siguiente manera:

El proceso de gestión consta de tres etapas principales: la formulación, la ejecución y la conclusión. En cada una de estas etapas debe realizarse la evaluación del programa, proyecto o actividad académica para decidir sobre su aprobación, refrendo y aval por parte de los responsables del proceso. (Universidad Nacional, 2010, p. 9)

Para esto posteriormente se darán recomendaciones y sugerencias que permitan presentar una propuesta para que las personas académicas tengan claro la naturaleza de la extensión, cómo esta aporta al desarrollo individual y colectivo de todas las personas participantes de las actividades, y cuál debe ser el proceso a seguir para establecer propuestas viables, con la aprobación respectiva, definiendo claramente el área de desarrollo.

\section{Metodología}

Esta investigación tomó como referencia la metodología híbrida de investigación, la cual incluye la combinación de métodos en un mismo estudio, como lo mencionan los autores Molina, López, Pereira, Pertusa y Tarí (2012). A partir de esta metodología se desarrollaron varias actividades, por ejemplo: 


\section{Análisis de textos sobre extensión}

Como parte de las actividades iniciales se consultaron textos relacionados con la temática de extensión, particularmente se consultó la normativa vigente de la Universidad Nacional, por ejemplo, el Estatuto Orgánico aprobado en el año 2015, los Lineamientos para la Gestión de Programas, Proyectos y Actividades Académicas, así como acuerdos emitidos por el Consejo Nacional de Rectores para la creación del Régimen de Sedes Interuniversitarias.

En este análisis también se consultaron libros y artículos referentes a la extensión universitaria.

\section{Estudio de experiencias docentes}

Para este caso se recopilaron las experiencias del personal académico de dos de los planes de estudio que se imparten en la Sede Interuniversitaria de Alajuela; la documentación de estas experiencias se realizó por medio de descripciones de cada académico o académica a cargo de los cursos.

\section{Entrevista a profundidad}

Para estudiar los detalles respecto a la creación y funcionamiento de la Sede Interuniversitaria de Alajuela se realizó una entrevista a la coordinadora académica de la Universidad Nacional en la Sede; como resultado de esta entrevista se obtuvo acceso a los documentos que dieron origen a la Sede Interuniversitaria. Asimismo, se facilitó información general sobre la forma de asignación del presupuesto que se otorga a las universidades instaladas en este espacio.

\section{Importancia y necesidad de la extensión en las Sedes Interuniversitarias}

En los últimos años se ha estudiado arduamente cómo las nuevas generaciones aprenden, socializan y se integran al mundo laboral, lo que lleva a cualquier docente a replantear y analizar si la metodología de enseñanza que utiliza en clase permite enrumbar al estudiantado no solo hacia el éxito académico, sino también hacia la adaptación con sus primeras experiencias laborales y hacia la comprensión de la realidad social. 
Revista Universidad en DiÁlogo • Vol. 8, N. ${ }^{\circ}$ 1, Enero-Junio, 2018, pp. 57-71

ISSN 2215-2849 • EISSN: 2215-4752

DOI: http://dx.doi.org/10.15359/udre.8-1.4

La plataforma de la extensión universitaria, que en su génesis permite la interacción entre lo aprendido y el entorno social, propicia que los y las estudiantes socialicen con su razón de ser profesional, en otras palabras, permite experimentar, comparar y replantear de forma vivencial con los proyectos de acercamiento a las comunidades.

La extensión se proyecta como una acción permanente por medio de la cual la institución aporta el conocimiento y la estrategia para que las comunidades, grupos y sectores se promuevan y avancen en su desarrollo. La extensión, a su vez, es el área académica que abstrae y produce conocimiento estratégico sobre la realidad de la sociedad, sus grandes aspiraciones, sus problemas, sus recursos y sus formas cómo se plantean y logran sus procesos de desarrollo humano y local (Ruiz, 2011).

Según el planteamiento anterior, la extensión permite alcanzar, mediante programas y actividades, entre otros eventos, un acercamiento del docente con diferentes entidades, tanto físicas como jurídicas, buscando una proyección integral de todos los actores que puedan promover y fomentar con una visión de desarrollo, llevando a la realidad prácticas enfocadas en procesos y fortalecimiento de temáticas administrativas, productivas, ambientales, así como generar diferentes habilidades en las personas involucradas que permitan fomentar la planificación y sistematización, estableciendo proyectos con una misión y visión, integrando a diferentes académicos y académicas de la Sede Interuniversitaria.

En este mismo texto se plantea que:

El proceso de la extensión universitaria genera espacio y oportunidades para el crecimiento de todos los participantes desde el momento mismo en que los académicos inician el contacto con las personas y las organizaciones que son sujeto de las acciones. Es importante tener presente esos diferentes espacios y oportunidades que se nos abren cuando trabajamos en los proyectos y programas de extensión (Ruiz, 2011).

Para el caso de la Universidad Nacional y las carreras que se imparten en la Sede Interuniversitaria, este contacto con las personas y las organizaciones ya se ha iniciado, y como resultado de este acercamiento solamente falta formalizar la creación de proyectos de extensión, puesto 
que en la actualidad no existen proyectos de esta naturaleza en ninguna de las carreras que se imparten por parte de la Universidad Nacional. Los resultados que se exponen a continuación corresponden a hechos enmarcados en los programas de los cursos y que no están vinculados a proyectos de extensión formalmente establecidos.

\section{Estado de situación de las acciones de extensión en la Sede Interuni- versitaria de Alajuela}

El propósito de creación de las Sedes Interuniversitarias es fundamental para el desarrollo de las zonas regionales; si bien la acción sustantiva de la docencia se ha ejecutado de manera satisfactoria desde su creación, existe el reto para todas las universidades públicas de impulsar por medio de la actividad docente la extensión, con proyectos que impacten en el desarrollo de las comunidades cercanas.

Además, las Sedes Interuniversitarias deben convertirse en el centro formador de profesionales con capacidad para impulsar el crecimiento de sus comunidades.

Sibien en la Sede Interuniversitaria deAlajuela no existen proyectos de extensión formalmente inscritos, como resultado de este artículo se presentan algunos de los esfuerzos que los académicos han realizado dentro de los programas de los cursos con el fin de emular buenas prácticas de acercamiento con la comunidad, no obstante, muy lejos de ser llamados extensión universitaria. La experiencia en campo ha engendrado en la Sede Interuniversitaria la necesidad de ir más allá de una gira o un aporte aislado a la sociedad, convirtiendo esfuerzos independientes en una extensión universitaria con impacto más tangible y un adecuado seguimiento y cierre de procesos.

Un ejemplo de esto son las soluciones empresariales que los y las estudiantes brindan a micro y pequeñas empresas de las provincias de Heredia y Alajuela en problemas cotidianos; un ejercicio que les permite a los estudiantes conocer de primera mano los problemas de los pequeños negocios, lo cual resulta en una experiencia muy enriquecedora en términos de aprendizaje. También, la siembra de árboles realizada por los estudiantes en octubre del 2016, en el cantón de San Isidro de Heredia, donde en coordinación con la Municipalidad se logró reforestar los alrededores del río Tures, con más de sesenta árboles nativos. Lamentablemente, la experiencia terminó ahí, el mantenimiento y 
Revista Universidad en DiÁlogo • Vol. 8, N. ${ }^{\circ}$ 1, Enero-Junio, 2018, pp. 57-71

ISSN 2215-2849 • EISSN: 2215-4752

DOI: http://dx.doi.org/10.15359/udre.8-1.4

continuidad del proyecto quedará sujeto a que las autoridades puedan contar con recursos para el mantenimiento y sostenimiento. Si se toman como referencia estos ejemplos para dimensionar la importancia de invertir en la extensión universitaria como una herramienta funcional para cerrar procesos planificados, permitiendo la continuidad con una sistematización oportuna de los proyectos por medio de resultados cuantitativos y cualitativos de las actividades, en definitiva se fortalecerá la relación universidad-comunidad a través de una adecuada comunicación.

Otro ejemplo valioso de la extensión universitaria está en la concientización que se logra generar en la población estudiantil con estos proyectos. En el curso Administración y Ambiente se realizó una gira para visitar el río Savegre, ubicado en San Gerardo de Dota, donde se compartió una charla con personas expertas que combinan la administración con la naturaleza y la comunidad.

En fin, estas pequeñas actividades logran un alto impacto en las personas jóvenes y en las comunidades y con certeza se puede afirmar que desarrollar la extensión universitaria potencializa las competencias blandas del estudiantado en relaciones interpersonales, trabajo en equipo, entre otros, así como la calidad de su aprendizaje. Tal como lo manifiesta Paulo Freire, "el primer aspecto que hay que destacar es que la práctica educativa es una dimensión necesaria de la práctica social" (1993, p. 73) y, por su parte, nuestro Estatuto Orgánico existe para que la comunidad estudiantil "disponga de oportunidades de acceso al estudio y de una formación universitaria de calidad" (2015, p. 27) coherente con los derechos del estudiantado.

Se evidencia la necesidad de avanzar integralmente, es el compromiso de la institución promover el pensamiento crítico de la población estudiantil, más allá de la teoría. Tal como lo manifiesta Alfredo Falero, "permite pasar del 'saber información' al 'saber pensar"' (2009, p. 13), de esta forma el involucramiento se vuelve palpable y dinámico a través de la extensión universitaria.

\section{Modelo de análisis administrativo para la incorporación de la exten- sión en zonas regionales}

Los esfuerzos independientes que realizan las personas académicas resultan en importantes plataformas para plantear formalmente proyectos de extensión en las Sedes Interuniversitarias y en consecuencia en las zonas regionales. La presentación de los antecedentes, la sensibilización y las 
prácticas llevadas a cabo por la Sede Interuniversitaria en cuanto al tema de extensión generan la necesidad de contar con una política claramente establecida de cuáles deben ser los pasos a seguir para llevar a la práctica en conjunto con el procedimiento administrativo adecuado a nivel interno.

Es por ello que a continuación se propone un modelo de análisis administrativo atinente a este tipo de acciones que fomentan una antesala de estudio para la propuesta de una política de extensión formal a nivel de la Universidad Nacional.

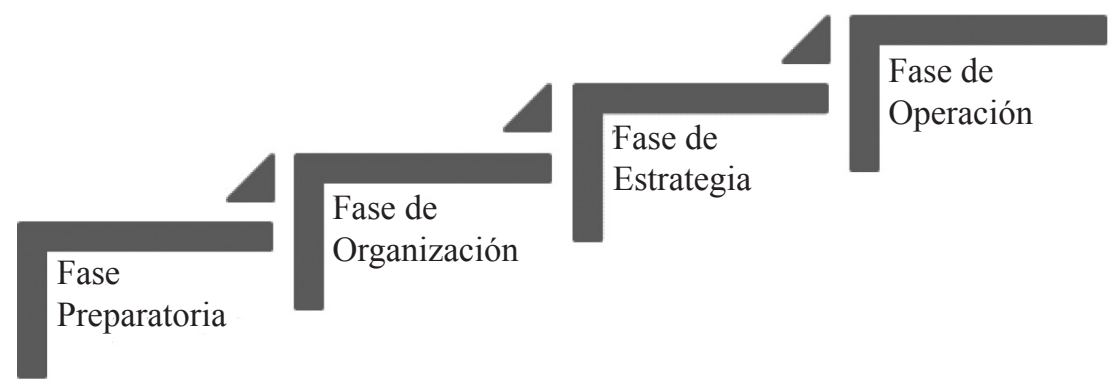

Figura 1. Fases de una política de extensión

Nota: S. A. (2014). Seminario Internacional sobre el Desarrollo Rural y Política Fiscal. Centroamérica 2021 hacia un Desarrollo Rural Equitativo IICA.

\section{Fase preparatoria}

En esta fase inicial se fomenta la práctica de las siguientes actividades:

1. Identificación de las organizaciones o grupos de interés de la zona donde se ubica la Sede Interuniversitaria: para establecer contactos en cada una de estas organizaciones para conocer las necesidades y expectativas de la comunidad respecto a la Universidad.

2. Capacitación al personal académico de la Sede Interuniversitaria: en la importancia de la extensión universitaria con el objetivo de motivar en los profesores y las profesoras la incorporación de esta acción en cada uno de los programas de los cursos.

3. Definir los ejes temáticos de interés con base en las siguientes áreas de desarrollo, definiendo el modelo a utilizar: 
Revista Universidad en Diálogo • Vol. 8, N. ${ }^{\circ} 1$, Enero-Junio, 2018, pp. 57-71

ISSN 2215-2849 • EISSN: 2215-4752

DOI: http://dx.doi.org/10.15359/udre.8-1.4

- Ambiente, conservación y manejo de los recursos naturales

- Desarrollo científico y tecnológico e innovación

- Tecnologías de información y comunicación

- Educación y desarrollo integral

- Gestión universitaria y emprendedurismo

- Humanismo, arte y cultura

- Salud y calidad de vida

- Sociedad y desarrollo humano

4. Sensibilización a los y las estudiantes: Contribuir por medio de la extensión en la formación de personas profesionales con un alto compromiso con el desarrollo social, interesadas en convertirse en promotoras del desarrollo en las comunidades de origen.

5. Definir la metodología que será utilizada para cada uno de los proyectos: donde como mínimo se exponga el problema planteado, el diagnóstico, los objetivos de la actividad, las conclusiones y las recomendaciones.

6. Defensa pública del anteproyecto: donde las personas involucradas en el proceso, ya sean académicas o estudiantes, desde el punto de partida de la presentación del proyecto para su aprobación, tengan una participación activa, donde darán a conocer aspectos generales del mismo, para su posterior revisión documental por parte de la Dirección de Área en conjunto con la Dirección de la Unidad Académica, donde se solventen aspectos de pertinencia y calidad del producto final que se puede obtener a través de la puesta en práctica del mismo.

\section{Fase de organización}

La Sede Interuniversitaria debe promover el intercambio académico estudiantil con instituciones, empresas o grupos de interés en torno a las disciplinas de las universidades participantes en la Sede. 
Es por ello que en la fase de organización se deben establecer fechas para la presentación de los proyectos, fechas de revisión y de aprobación de los mismos. Fomentando no solo la práctica, sino la ejecución adecuada de la extensión de acuerdo a la normativa actual, en cuanto a la forma en que se presentan los documentos actualmente para dichas gestiones.

\section{Fase de estrategia}

Se debe establecer en el presupuesto operativo anual de la Sede Interuniversitaria la designación de los recursos necesarios para la apertura de proyectos de extensión, garantizando la dotación de los recursos por parte del Consejo Nacional de Rectores para las jornadas académicas dirigidas a proyectos de extensión universitaria, en cada una de las universidades participantes en la Sede Interuniversitaria.

Para ello es necesario contar con un planteamiento para la solicitud de recursos de los posibles proyectos de extensión que se estarán llevando a cabo de manera anual, identificando y cuantificando la cantidad y calidad de los recursos que serán necesarios para cada uno de los proyectos.

Se llevará a cabo, mediante una herramienta como Gráfica de Gantt, un control de las actividades a realizar, así como de las personas responsables y las fechas de inicio y conclusión de cada una de las fases. Tomando en cuenta la táctica y la logística correspondiente en cada uno de los pasos.

\section{Fase de operación}

En esta fase será necesaria la aplicación de métodos de supervisión y control, así como instrumentos e indicadores de medición, donde se harán evaluaciones parciales del proyecto, alineando siempre los pre-resultados con los objetivos iniciales planteados. Promoviendo la corrección de aspectos que no estén alineados en el camino del proyecto, dando una retroalimentación continua a cada una de las personas involucradas.

\section{Recomendaciones}

Las Sedes Interuniversitarias se visualizan como un promotor de enlaces entre la comunidad y los grupos de interés, tales como organizaciones, empresas y otros grupos de interés de la zona donde su ubica la Sede, 
Revista Universidad en DiÁlogo • Vol. 8, N. ${ }^{\circ}$ 1, Enero-Junio, 2018, pp. 57-71

ISSN 2215-2849 • EISSN: 2215-4752

DOI: http://dx.doi.org/10.15359/udre.8-1.4

por medio de acuerdos y convenios de cooperación; es por ello que se deben tomar medidas para que las políticas de extensión se conviertan en una herramienta que facilite el impulso de las micro, pequeñas y medianas empresas en las zonas regionales.

Se propone establecer vínculos con grupos de interés de las comunidades en torno a las disciplinas de las universidades participantes de las Sedes Interuniversitarias. También se aconseja contribuir por medio de la extensión en la formación de profesionales con un alto compromiso con el desarrollo social, que anhelen convertirse en personas promotoras del desarrollo en sus comunidades de origen.

Se recomienda promover el emprendimiento en la población estudiantil de la Sede Interuniversitaria a través de los programas de los cursos en los diferentes planes de estudio, con el objetivo de crear el perfil del o de la estudiante extensionista con condiciones para convertirse en un generador de empleo en las zonas regionales y de esta manera cumplir con el propósito de "f. Maximizar el aprovechamiento de los recursos destinados al Sistema Universitario Estatal en beneficio de las poblaciones regionales y promover la permanencia de los estudiantes en sus comunidades"

Garantizar en el presupuesto operativo anual de cada Sede Interuniversitaria la asignación de los recursos necesarios para la apertura de proyectos de extensión.

Fomentar en los planes de estudio de las Sedes Interuniversitarias el eje del emprendimiento en la población estudiantil con el objetivo de crear el perfil del estudiantado que generará empleo en las zonas donde se ubiquen las Sedes.

Y por último impulsar en las instalaciones de la Sede Interuniversitaria la programación de actividades que involucren la participación de la comunidad, motivando principalmente la incorporación de los grupos en desventaja económica y social en la zona regional. 


\section{Referencias}

Consejo Nacional de Rectores (2007). Acuerdo CNR-106-07. San José, Costa Rica.

Falero, A. (2009). Universidad en movimiento: 2009. Universidad y pensamiento crítico. Debates y memorias del X Congreso Iberoamericano de Extensión Universitaria. Recuperado de http://www.documentos. una.ac.cr/handle/unadocs/8132

Freire, P. (1993). Política y educación. Madrid: Siglo XXI. Recuperado de http://www.documentos.una.ac.cr/handle/unadocs/8132.

Molina, J., López, M., Pereira, J., Pertusa, E. y Tarí, J. (2012). Métodos híbridos de investigación y dirección de empresas: ventajas e implicaciones. Recuperado de http://www.elsevier.es/cede.

Newstrom, J. (2011). Comportamiento humano en el trabajo (13 ${ }^{\mathrm{a}}$. ed.). México: Editorial McGraw Hill.

Ruiz, R. (2011). Mirando el pasado, soñando el futuro. Extensión: transformaciones vitales en la relación Universidad-Comunidad. Universidad en Diálogo, 1(1), 13-32.

S. A. (2014). Seminario Internacional sobre Desarrollo Rural y Politica Fiscal. Centroamérica 2021 hacia un Desarrollo Rural Equitativo. Sistema de Integración Centroamericana (SICA). Recuperado de http:/www.documentos.una.ac.cr/bitstream/handle/unadocs/8084/ Enfoque-territorial-taller-ICEFI-2014

Universidad Nacional (2010). Lineamientos para la Gestión de Programas, Proyectos y Actividades. Vicerrectoría de Docencia. Recuperado de http://documentos.una.ac.cr/handle/unadocs/1793

Universidad Nacional (2011). Gestión de proyectos. Heredia, Costa Rica. Recuperado de http://www.extension.una.ac.cr/index.php

Universidad Nacional (2015). Estatuto Orgánico. Heredia, Costa Rica. 